\title{
Impact of Percentage of Positive Biopsy Cores on Biochemical Outcome in Patients Treated With Low-Dose Rate (Iodine-125) Brachytherapy for Prostate Cancer
}

\author{
C. Yuen ${ }^{* 1}$, T. Hossack ${ }^{1}$, A.M. Haynes ${ }^{3}$, R.A. Pe Benito ${ }^{3}$, J. Matthews ${ }^{1}$, G. Fogarty ${ }^{2}$, R. Jagavkar ${ }^{2}$, \\ P. Brenner ${ }^{1}$ and P. Stricker ${ }^{1}$ \\ ${ }^{I}$ St Vincent's Clinic, Department of Urology, Darlinghurst, NSW, Australia \\ ${ }^{2}$ St Vincent's Clinic Department of Radiation Oncology, Darlinghurst, NSW, Australia \\ ${ }^{3}$ Garvan Institute of Medical Research, Darlinghurst, NSW, Australia
}

\begin{abstract}
To investigate the significance of the percentage of positive biopsy cores (PPBCs) in predicting the biochemical outcome in patients with clinically localised prostate cancer undergoing low-dose rate brachytherapy (LDRB).

- A total of 326 consecutive patients underwent LDRB between February 1997 and January 2007. The cohort consisted of $68.7 \%, 30.4 \%$ and $0.9 \%$ of low-, intermediate- and high-risk groups respectively as defined by the D'Amico classification. Patients were stratified according to PPBCs $(<35 \%, 35-50 \%,>50 \%)$.

- Of the 326 men, 316 (97\%) met the study criteria and were included in the analysis. The median follow-up was 66.3 (12.9-147.5) months. Thirty-eight men (12\%) developed evidence of biochemical relapse as defined by the Phoenix definition at a median of 44.5 (4-133) months. The overall 5-year and 10-year biochemical relapse-free rate (BRFR) was $91.9 \%$ (95\% CI $87.9 \%$ to $94.6 \%$ ) and $76.9 \%$ (95\% CI $66 \%$ to $84 \%)$ respectively.

- On univariate analysis, Gleason score $(\mathrm{p}=0.03)$, D'Amico risk groups $(\mathrm{p}=0.004)$ and PPBCs $(\mathrm{p}=0.001)$ were significant predictors of biochemical failure. In the multivariate model, the PPBCs $(p=0.0006)$ and pretreatment PSA $(p=0.004)$ were the only variables that predicted for biochemical failure. The 5-year biochemical relapse-free rate was $93.5 \%, 93.0 \%$ and $76.5 \%$ for the $\mathrm{PPBCs}<35 \%, 35 \%-50 \%$ and $>50 \%$ respectively $(\mathrm{p}=0.001)$.

Conclusions: - The PPBC is an important independent predictor of the 5-year biochemical relapse-free survival after LDRB. This finding suggests that patients with high volume tumour are at increased risk of biochemical failure with LDRB.
\end{abstract}

Keywords: Prostate malignancy, localised prostate cancer, interstitial brachytherapy, low-dose rate brachytherapy, percentage of positive biopsy cores, biochemical outcome, biochemical relapse-free survival.

\section{INTRODUCTION}

Prognostic features for clinically localised prostate cancer have been extensively studied. Pre-treatment PSA, biopsy Gleason score and clinical $\mathrm{T}$ stage have each been shown to be independent predictors of biochemical progression-free survival after radical prostatectomy, external beam radiotherapy (EBRT), or brachytherapy [1-3]. In addition, studies have demonstrated a correlation between percentage of positive biopsy cores (PPBC) and biochemical failure, as well as prostate cancer specific mortality, for patients treated with either surgery or radiotherapy [4-13]. For instance D' Amico and colleagues $[5,6]$ reported that percentage of positive biopsies was predictive of the biochemical outcome after

*Address correspondence to this author at the St Vincent's Clinic, Department of Urology, Darlinghurst, NSW, Australia; Tel: +61 28382 6960; Fax: +61 28324 6448; E-mails: carloyuen@hotmail.com; cyuen@stvincents.com.au either radical prostatectomy or 3-dimensional conformal EBRT.

Although recently the PPBC have been shown to have particular predictive value, there are limited reports describing its prognostic role after brachytherapy [9-12]. In this study, we investigate the significance of PPBC in predicting biochemical outcome in patients with clinically localised prostate cancer stratified into low, intermediate and high risk groups using the D'Amico classification [3].

\section{MATERIALS AND METHODOLOGY}

\section{Patient Population}

The study cohort consists of three hundred twenty-six consecutive men treated at St Vincent's Private Hospital (Darlinghurst, NSW 2010, AUS) between Feb 1997 and Jan 2007. The implantation cut off date was selected to allow for 
3 years of minimum potential follow-up. Institutional research ethics board approval was obtained for this study.

All men had clinically localised prostate cancer confirmed on biopsy. Ultrasound guided transrectal or transperineal biopsies were performed depending on the surgeon's preference, with a minimum of 6 cores sampled. The PPBC was obtained by dividing the total number of cores with prostate cancer by the total number of cores obtained.

Clinical workup before treatment included a history and physical examination, digital rectal examination, biopsy and prostate-specific antigen (PSA) measurement. Further investigations, such as a computerized axial tomography (CT) or bone scan, were obtained as clinically indicated. Patients were staged according to 2002 American Joint Committee on Cancer guidelines [14]. PSA measurements were obtained using standard assays at several different laboratories, depending on patient location.

\section{Treatment}

All patients underwent transperineal ultrasound-guided permanent Iodine-125 seeds brachytherapy for clinically organ-confined prostate cancer. In patients with prostate gland larger than $50 \mathrm{cc}$, androgen deprivation therapy (luteinizing hormone-release hormone agonist or anti-androgen) was used 3-4months before implantation to achieve cytoreduction. In some patients, hormone manipulation was commenced by the referring clinician before assessment for brachytherapy. In all patients, hormones were discontinued after implantation so that the duration of hormone manipulation was no more than 6 months in total.

LDRB was performed jointly with the radiation oncologist, physicist and urologist under general or spinal anaesthesia. The minimum peripheral dose (MPD) of 145 Gy was prescribed to cover at least $98 \%$ of the planned target volume according to Task Group 43 [15]. All plans used modified peripheral loading pattern with relative periurethral sparing, with the urethral dose limited to less than $150 \%$ of the MPD. At implantation, iodine- 125 seeds were placed in the prostate using real time transrectal ultrasound and fluoroscopic guidance, as described previously by the Seattle group [16].

\section{Postimplant Dosimetric Assessment}

Postimplant dosimetric assessment was performed on all LDRB patients from Feb 2000 onwards. Prior to this dosimetric assessment was not routinely performed. A total of 266 of $316(84.2 \%)$ underwent CT imaging of the pelvis according to a protocol using $3-\mathrm{mm}$ slices. The location of each seeds was determined and the CT images were used to analyse the dosimetric data using commercially available software. We report the minimal dose received by $90 \%$ of the target volume (D90), as recommended by the American Brachytherapy Society [17].

\section{Follow-Up}

The median follow-up was 66.3 (12.9-147.5) months for the entire cohort. PSA failure was defined by the RTOGASTRO, Phoenix Consensus Conference definition: an increase by $\geq 2 \mathrm{ng} / \mathrm{mL}$ greater than the nadir PSA level [18].
Patients without biochemical relapse were censored at death or last follow-up. All biochemical relapses identified in the prospective database were also verified by retrospective chart reviews. Patients who met criteria for biochemical relapse but had a subsequent decrease in PSA level without intervention to a new nadir of $0.5 \mathrm{ng} / \mathrm{mL}$ or less were classified as a benign bounce in PSA level and not coded as biochemical relapse.

\section{Statistical Methods}

A Cox regression univariate analysis was performed initially to determine whether PPBCs, pretreatment PSA, clinical T stage, Gleason score, dosimetry (D90), and D'Amico classification predicted the likelihood of biochemical failure after LDRB. In the multivariate analysis, the clinical variables of PPBCs, Gleason score, clinical $T$ stage and pretreatment PSA were examined. The patients were stratified according to the PPBC using the breakpoints of $<35 \%, 35 \%$ $50 \%$, and $>50 \%$. Biochemical relapse-free survival was estimated using the actuarial method of Kaplan and Meier (Statistical Analysis Systems, version 9.1.3, SAS Institute, Cary, NC) and was stratified into the PPBC subgroups of $<35 \%, 35 \%-50 \%$, and $>50 \%$. The log-rank test was used to evaluate the difference in survival curves.

\section{RESULTS}

Of the 326 men, 316 (97\%) met study criteria and were included in the analysis. The pre-treatment characteristics are provided in Table 1. The median pre-treatment PSA level was $7.25 \mathrm{ng} / \mathrm{mL}(1.1-21.6)$ and 217 (68.7\%) of the 316 men had non-palpable disease. The median number of biopsies was 8 (range 6-30, mean 8.70). The cohort consisted of $68.7 \%, 30.4 \%$ and $0.9 \%$ of low-, intermediate- and high-risk groups respectively as defined by the D'Amico classification. The median follow-up was 66.3 months (range 12.9147.5). The number of patients who had PPBCs of $<35 \%$, $35 \%-50 \%$, $>50 \%$ was $217(68.7 \%), 72(22.8 \%)$, and 27 $(8.5 \%)$, respectively.

Androgen deprivation therapy (ADT) was prescribed in $88(27.8 \%)$ patients prior to implantation. Of the men that received ADT, $46(52.3 \%)$ and $42(47.7 \%)$ were in the low and intermediate D'Amico risk groups respectively. The 5year biochemical relapse-free rate for patients undergoing brachytherapy alone or with pre-implant hormone manipulation were $93 \%$ (95\% CI $89 \%$ to $96 \%$ ) and $89 \%$ (95\% CI $79 \%$ to $94 \%$ ) respectively $(\mathrm{p}=0.32)$.

Dosimetry (D90) was recorded in 266 patients. D90 $\geq 140$ Gy was achieved in 124 patients and the median D90 was 138.5 Gy. Dosimetry did not predict for biochemical relapse on cox regression analysis and was therefore not included in the multivariate analysis.

A total of $38(12.0 \%)$ men developed evidence of biochemical recurrence during the entire follow-up period at a median of 44.5 months (4-133). Of the 38 men, $14(36.8 \%)$ had local recurrence confirmed on biopsy and 5 (13.2\%) developed distant metastatic disease confirmed on imaging. Of the remaining men with biochemical failure there was no documented local recurrence or metastatic disease. Six patients underwent salvage therapy for local recurrence. 
Table 1. Patient Demographics and Characteristics $(n=316)$

\begin{tabular}{|l|c|}
\hline Variable & Value \\
\hline \hline Age & \\
Median & 63.8 \\
Range & $47-81$ \\
\hline T stage & \\
T1a-b & $5(1.6)$ \\
T1c & $212(67.1)$ \\
T2a & $73(23.1)$ \\
T2b & $24(7.6)$ \\
T2c & $2(0.6)$ \\
\hline Gleason score & \\
$\leq 6$ & $271(85.8)$ \\
7 & $45(14.2)$ \\
$\geq 8$ & $0(0)$ \\
\hline Pretreatment PSA & \\
$\leq 10$ & $268(84.8)$ \\
$11-20$ & $47(14.9)$ \\
$>20$ & $1(0.3)$ \\
\hline PPBCs & \\
$<35 \%$ & $217(68.7)$ \\
$35-50 \%$ & $72(22.8)$ \\
$>50 \%$ & $27(8.5)$ \\
\hline D90 (Gy) & 138.45 \\
Median & $64.5-173.8$ \\
Range & \\
\hline Follow-up (mth) & \\
Median & CI $87 \%$ - \\
Range & \\
\hline 5 -yr biochemical relapse-free survival rate & \\
& \\
\hline
\end{tabular}

Fig. (1a) shows the Kaplan-Meier biochemical relapsefree survival for the entire cohort. The 5-year and 10-year BRFR were $91.9 \%(95 \%$ CI $87.9 \%$ to $94.6 \%)$ and $76.9 \%$ (95\% CI 66\% to 84\%) respectively. Fig. (1b) shows the Kaplan-Meier biochemical relapse-free survival for low-, and intermediate- subgroups according to the D'Amico classification.

The results of univariate analysis for BRFR are shown in Table 2. Four variables were significantly associated with BRFR: PPBCs $(p=0.001)$, pre-treatment PSA level $(<0.001)$, D'Amico risk groups $(\mathrm{p}=0.004)$ and Gleason score $(\mathrm{p}=0.03)$. The 5-year biochemical relapse-free rate was 93.5\%, 93.0\% and $76.5 \%$ for the PPBCs $<35 \%, 35 \%-50 \%$ and $>50 \%$ respectively (Fig. 2). In the multivariate analysis, the PPBCs $(p=0.0006)$ and pretreatment PSA $(p=0.004)$ were the only variables that predicted for biochemical relapse-free survival.

\section{DISCUSSION}

In the present study, the prognostic significance of PPBC and several disease-specific variables was investigated in men with predominantly low- and intermediate-risk localised prostate cancer treated with LDRB alone. We found the
PPBCs to be a powerful predictor of biochemical relapsefree survival. Particularly in those with PPBCs $>50 \%$, this strongly predicts for the likelihood of biochemical failure. The 5-year BRFR for PPBC $<50 \%$ was $93.0 \%$ versus $76.5 \%$ for $\mathrm{PPBC}>50 \%(\mathrm{p}=0.0006)$.

This finding is supported by Rossi et al. [9] who recently demonstrated a correlation between tumour volume, as determined by the PPBCs, and the time to PSA failure in pa-

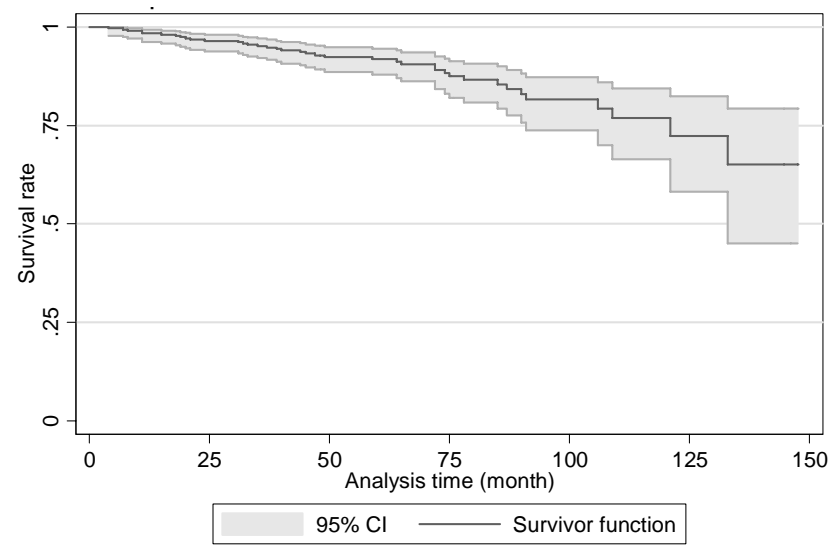

Fig. (1a). Kaplan-Meier survival estimate for the entire cohort.

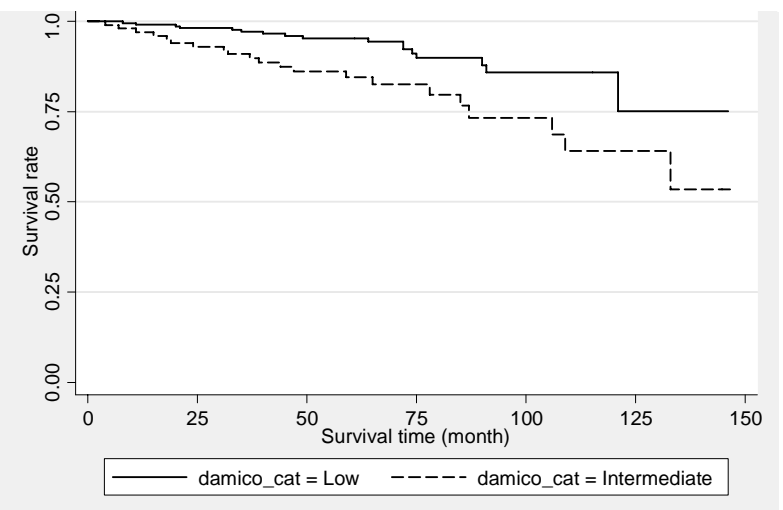

Fig. (1b). Kaplan-Meier survival estimate by D'Amico classification.

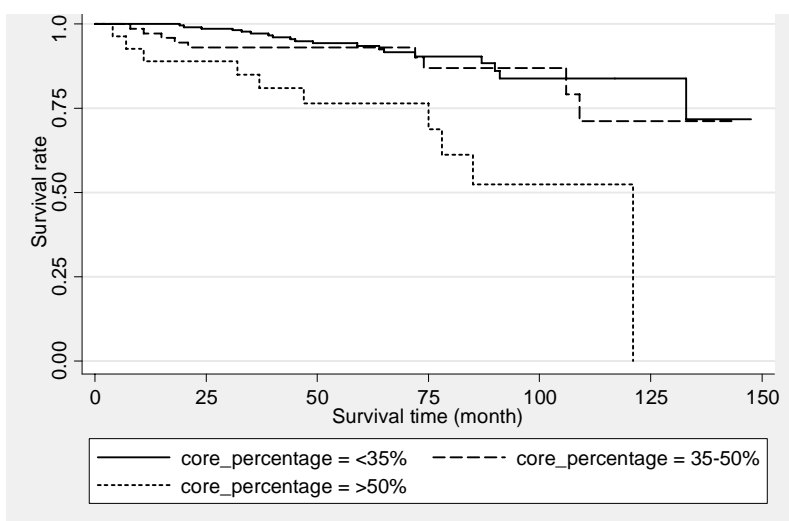

Fig. (2). Kaplan-Meier survival estimate by core percentage (PPBC). 
Table 2. Univariate and Multivariate Analysis of Predictors of Biochemical Failure after LDRB

\begin{tabular}{|lll|}
\hline Variable & $\begin{array}{l}\text { Hazard } \\
\text { ratio }\end{array}$ & P value \\
\hline Univariate analysis & & \\
PPCBs ( $<35 \%, 35 \%-50 \%,>50 \%)$ & 2.0 & 0.001 \\
D'Amico (low vs intermediate) & 2.6 & 0.004 \\
Gleason score ( $\leq 6$ vs $>6$ ) & 2.2 & 0.03 \\
T stage (T1 vs T2) & 1.05 & 0.89 \\
Pretreatment PSA (continuous) & 1.16 & $<0.001$ \\
D90 (continuous) & 0.98 & 0.25 \\
Multivariate analysis & & \\
PPCBs (<35\%, 35\%-50\%, >50\%) & 4.40 & 0.0006 \\
Gleason score ( $\leq 6$ vs $>6$ ) & 1.49 & 0.33 \\
T stage (T1 vs T2) & 0.83 & 0.62 \\
Pretreatment PSA (continuous) & 1.12 & 0.004 \\
\hline
\end{tabular}

tients undergoing brachytherapy for clinically localised prostate cancer. They reported that patients with $<50 \%$ positive biopsy cores had a 5-year estimated biochemical-free survival rate of $95 \%$ and patients with $>50 \%$ positive biopsy cores had a 5-year biochemical-free survival rate of $63 \%$.

Rossi et al. suggested that PPBC $>50 \%$ is a surrogate for a more malignant phenotype and there are several reports supporting this. The PPBCs has been associated with adverse pathologic findings in radical prostatectomy specimens [19,20]. Quinn et al. [19] reviewed the pretreatment characteristics of 696 men with localised prostate cancer treated by radical prostatectomy. They found that increasing PPBCs predicted for extracapsular extension, positive surgical margins, seminal vesicle invasion, and disease recurrence. Similar results have been reported by Freedland et al. [8] and Sebo et al. [20].

The relationship between PPBCs and biochemical failure has also been examined by Merrick et al. [10]. They reported on 262 patients who underwent brachytherapy and found a significant trend for PSA failure with increasing PPBC but did not find statistical significance in predicting 5-year biochemical relapse-free survival when stratified by low-, intermediate-, and high-risk disease groups. Statistical significance was achieved, however, in a later study that included 7 years of follow-up and recruitment of 150 additional patients [21].

In contrast, Pe et al. [12] retrospectively reviewed 360 patients with low-risk prostate cancer and evaluated the prognostic significance of PPBCs in predicting the time to biochemical relapse for patients receiving either brachytherapy or 3D-CRT. The pre-treatment PSA level, PPBCs, or treatment modality was not found to be predictive of the time to biochemical failure. The author concluded that this might have been influenced by both the slow rate of biochemical recurrence in the low-risk prostate cancer population, as well as the relatively few number of cases of highvolume disease. They recommended additional studies with longer follow-up and recruitment of patients with highvolume disease are needed.

Several reports examining the PPBCs in patients undergoing either radical prostatectomy or radiotherapy have shown PPBC to be an independent predictor of freedom from biochemical relapse [3,5,7]. In addition, D'Amico et al. [13] found that the PPBCs was a significant predictor of prostate cancer-specific mortality (PCSM) in those who underwent 3D-conformal radiation therapy (CRT) for low or favourable intermediate-risk cancers. In particular, they showed that for patients with $\geq 50 \%$ positive biopsy cores, the relative risk of PCSM was 6-12 fold greater compared with men with $<50 \%$ positive cores.

The current study and the reports outlined support PPBC $>50 \%$ as an important predictor for biochemical failure in patients undergoing LDRB for localised prostate cancer. The strength of the study is its sample size and the length of followup. The limitations include sampling bias due to lack of standardisation of biopsy technique, hence may influence the calculation of PPBCs. Furthermore, it is unclear whether short term neoadjuvant hormone manipulation have a significant impact on biochemical control.

\section{CONCLUSION}

The PPBC is an important independent predictor of biochemical relapse-free survival after LDRB. These findings suggest that patients with PPBC greater than 50\% are at increase risk of biochemical failure with LDRB as monotherapy.

\section{ACKNOWLEDGEMENTS}

Prostate cancer research at the Garvan Institute/St Vincent's Hospital is supported in part by grants from the National Health and Medical Research Council of Australia, Cancer Institute New South Wales, Prostate Cancer Foundation of Australia, Australia Cancer Research Foundation, St Vincent's Prostate Cancer Centre, the RT Hall Trust and the Petre Foundation.

\section{CONFLICT OF INTEREST}

None Declared

\section{REFERENCES}

[1] Kattan MW, Easthan JA, Stapleton AMF, et al. A preoperative nomogram for disease recurrence following radical prostatectomy for prostate cancer. J Natl Cancer Inst 1998; 90: 766-71.

[2] D'Amico AV, Whittington R, Kaplan I, et al. Equivalent biochemical failure free survival after external beam radiation therapy or radical prostatectomy in patients with a pretreatment prostate specific antigen >4-20 ng/mL. Int J Radiat Oncol Biol Phys 1997; 37 : 1053-8.

[3] D'Amico AV, Whittington R, Malkowicz SB, et al. Biochemical outcome after radical prostatectomy, external beam radiation therapy or interstitial radiation therapy for clinically localized prostate cancer. JAMA 1998; 280: 969-74.

[4] Sebo TJ, Bock BJ, Cheville JC, et al. The percent of cores positive for cancer in prostate needle biopsy specimen is strongly predictive of tumor stage and volume at radical prostatectomy. J Urol 2000; 163: 174-8.

[5] D'Amico AV, Whittington R, Malkowicz SB, et al. Clinical utility of the percentage of positive prostate biopsies in defining biochem- 
ical outcome after radical prostatectomy for patients with clinically localised prostate cancer. J Clin Oncol 2000; 18: 1164-72.

[6] D'Amico AV, Whittington R, Malkowicz SB, et al. Clinical utility of percent-positive prostate biopsies in predicting biochemical outcome after radical prostatectomy or external beam radiation therapy for patients with clinically localized prostate cancer. Mol Urol 2000; 4: 171-5.

[7] D'Amico AV, Schultz D, Silver, et al. The clinical utility of the percent of positive prostate biopsies in predicting biochemical outcome following external beam radiation therapy for patients with clinically localized prostate cancer. Int J Radiat Oncol Biol Phys 2001; 49: 679-84.

[8] Freeland SJ, Aronson WJ, Terris MK, et al. Percent of prostate needle biopsy cores with cancer is a significant independent predictor of prostate specific antigen recurrence following radical prostatectomy: results from the SEARCH database. J Urol 2003; 169: 2136-41.

[9] Rossi PJ, Clark PE, Papagikos MA, et al. Percentage of positive biopsies associated with freedom from biochemical recurrence after low-dose-rate prostate brachytherapy alone for clinically localized prostate cancer. Urology 2006; 67: 349-53.

[10] Merrick GS, Butler WM, Galbreath RW, et al. Relationship between percent positive biopsies and biochemical outcome after permanent interstitial brachytherapy for clinically organ-confined carcinoma of the prostate gland. Int J Radiat Oncol Biol Phys 2002; 52:664-73

[11] Merrick GS, Butler WM, Wallner KE, et al. Prognostic significance of percent positive biopsies in clinically organ-confined prostate cancer treated with permanent prostate brachytherapy with or without supplemental external beam radiation. Cancer J 2004; 10 : 54-60.

[12] Pe ML, Trabulsi EJ, Kedika R, et al. Effect of percentage of positive prostate biopsy cores on biochemical outcome in low-risk PCa treated with brachytherapy or 3D-CRT. Urology 2009; 73:1328-34.

[13] D'Amico AV, Renshaw AA, Cote C, et al. Impact of the percentage of positive cores on prostate cancer-specific mortality for pa- tients with low or favourable intermediate-risk disease. J Clin Oncol 2004; 22: 3726-32.

[14] Greene FL, Page DL, Fleming ID, et al. For the american joint committee on cancer. manual for staging cancer. $6^{\text {th }}$ ed. New York: Springer-Verlag 2002; pp. 337-46.

[15] Nath R, Anderson LL, Luxton G, et al. Dosimetry of interstitial brachytherapy sources: Recommendations of the AAPM Radiation Therapy Committee Task Group No. 43. Med Phys 2000; 22: 20934.

[16] Blasko JC, Mate T, Sylvester JE, et al. Brachytherapy for carcinoma of the prostate: Techniques, patient selection, and clinical outcomes. Semin Radiat Oncol 2002; 12: 81-94.

[17] Nag S, Bice W, DeWyngaert $\mathrm{K}$, et al. The American Brachytherapy Society recommendations for permanent prostate brachytherapy postimplant dosimetric analysis. Int J Radiat Oncol Biol Phys 2000;46: 221-30.

[18] Roach M, Hanks G, Thames H, et al. Defining biochemical failure following radiotherapy with or without hormonal therapy in men with clinically localized prostate cancer: recommendations of the RTOG-ASTRO, Phoenix consensus conference. Int J Radiat Oncol Biol Phys 2006; 65: 965-74.

[19] Quinn DI, Henshall SM, Brenner PC, et al. Prognostic significance of preoperative factors in localized prostate carcinoma treated with radical prostatectomy: importance of percentage of biopsies that contain tumour and the presence of biopsy perineural invasion. Cancer 2003; 97: 1884-93.

[20] Sebo TJ, Cheville JC, Riehle DL, et al. Predicting prostate carcinoma volume and stage at radical prostatectomy by assessing needle biopsy specimens for percent surface area and cores positive for carcinoma, perineural invasion. Gleason score, DNA ploidy and proliferation and preoperative serum prostate specific antigen: a report of 454 cases. Cancer 2001; 91: 2196-204.

[21] Merrick GS, Butler WM, Wallner KE, et al. Prognostic significance of percent positive biopsies in clinically organ-confined prostate cancer treated with permanent prostate brachytherapy with or without supplemental external beam radiation. Cancer J 2004; 10 : 54-60.

Received: April 22, 2012

Revised: May 10, 2012

Accepted: May 11, 2012

(C) Yuen et al.; Licensee Bentham Open.

This is an open access article licensed under the terms of the Creative Commons Attribution Non-Commercial License (http://creativecommons.org/licenses/ by-nc/3.0/) which permits unrestricted, non-commercial use, distribution and reproduction in any medium, provided the work is properly cited. 